\title{
DESCRIÇÃO DO PERFIL EPIDEMIOLÓGICO DA POPULAÇÃO INFECTADA COM HẢNSENÍASE NOS MUNICÍPIOS DE CUIABÁ E VÁRZEA GRANDE - MATO GROSSO
}

\author{
DESCRIPTION OF THE EPIDEMIOLOGICAL PROFILE OF THE POPULA- \\ TION INFECTED WITH HANSENIASIS IN CUIABÁ AND VÁRZEA GRANDE - \\ MATO GROSSO
}

Lucas Rangel Antunes Maciel ${ }^{*}$, Caio Leal Carvalho, Nathália Saboia Campos Borbon Novis Neves, Maria Paula Miranda Ferreira de Alencar Carvalho, Marcelo Rosa Guazina, Giovanna Sulzbacher Borghetti, Rosa Maria Elias.

Universidade de Cuiabá, Cuiabá, MT, Brasil.

*lucasrmaciel@hotmail.com

\section{RESUMO}

A hanseníase é uma doença infecto-contagiosa causada pelo Mycobacterium leprae, com evolução lenta e diversas formas clínicas associadas com o tipo de resposta do paciente ao $\mathrm{M}$. leprae. As manifestações clínicas são os sinais e sintomas dermatoneurológicos como lesões na pele e nos nervos periféricos, o que pode levar a vários prejuízos de esferas social e econômica. Uma característica epidemiológica é sua alta infectividade e baixa patogenicidade, ou seja, infecta muitas pessoas mas poucas adoecem. $O$ homem é considerado a única fonte de infecção, sendo que o contágio dá-se através de um bacilífero, não tratado, que elimina o Bacilo de Hansen para o meio exterior pelo trato respiratório, contagiando pessoas susceptíveis, através de um contato direto. Através desse trabalho, verificamos o número de notificações e descrevemos o perfil epidemiológico dos casos de Hanseníase na população dos municípios de Cuiabá e Várzea Grande, no estado do Mato Grosso. O fizemos através de um estudo transversal descritivo, com dados do Sistema de Informação de Agravos de Notificação (Sinan) no Departamento de Informática do Sistema Único de Saúde DATASUS referentes ao período entre 2000 a 2018 sobre as notificações de casos confirmados de Hanseníase, em população de 0 a 65+ anos de idade, nos municípios de Cuiabá e Várzea Grande, localizados no estado de Mato Grosso. Foram incluídos neste estudo as variáveis sociodemográficas como idade, sexo e dados clínicos como o tipo de entrada da doença, a forma clínica e evolução dos casos. Foram notificados 13.989 casos totais de Hanseníase, representando 21,9\% de todos os casos registrados em Mato Grosso durante 0 período, entre residentes de Cuiabá $(69,9 \%)$, do sexo masculino $(56,2 \%)$, com idade entre $65+$ anos (9,3\%). A maioria das notificações foram de casos confirmados da forma clínica dimorfa (37\%), com confirmação clínico-laboratorial (90\%), com evolução para a cura da doença $(66,8 \%)$. A menor taxa de ocorrência da doença foi registrada no ano de 2016 (3,8\%). Visto isso, as ações voltadas para o diagnóstico precoce da Hanseníase, um dos principais desafios de saúde pública no estado de Mato Grosso, começam a mostrar efetividade para um tratamento adequado desta patologia em Cuiabá e Várzea Grande, municípios que se encontram no estado com maior índice da hanseníase no Centro-Oeste, 
o que os coloca em uma posição de risco para tal doença. A doença ainda é um problema de saúde pública, necessitando maior vigilância resolutiva.

Palavras-chave: Dermatopatias. Doenças transmissíveis. Epidemiologia analítica. Hanseníase. Mycobacterium leprae. 